\title{
MULTIPLICITIES, INVARIANT SUBSPACES AND AN ADDITIVE FORMULA*
}

\author{
ARUP CHATTOPADHYAY ${ }^{1}$, JAYDEB SARKAR ${ }^{2}$ AND SRIJAN SARKAR ${ }^{3}$ \\ ${ }^{1}$ Department of Mathematics, Indian Institute of Technology Guwahati, Guwahati \\ 781039, India (arupchatt@iitg.ac.in, 2003arupchattopadhyay@gmail.com) \\ ${ }^{2}$ Statistics and Mathematics Unit, Indian Statistical Institute, 8th Mile, Mysore Road, \\ Bangalore 560059, India (jay@isibang.ac.in, jaydeb@gmail.com) \\ ${ }^{3}$ Department of Mathematics, Indian Institute of Science, Bangalore 560012, India \\ (srijans@iisc.ac.in, srijansarkar@gmail.com)
}

(Received 02 June 2020; first published online 30 April 2021)

Abstract Let $T=\left(T_{1}, \ldots, T_{n}\right)$ be a commuting tuple of bounded linear operators on a Hilbert space $\mathcal{H}$. The multiplicity of $T$ is the cardinality of a minimal generating set with respect to $T$. In this paper, we establish an additive formula for multiplicities of a class of commuting tuples of operators. A special case of the main result states the following: Let $n \geq 2$, and let $\mathcal{Q}_{i}, i=1, \ldots, n$, be a proper closed shift co-invariant subspaces of the Dirichlet space or the Hardy space over the unit disc in $\mathbb{C}$. If $\mathcal{Q}_{i}^{\perp}, i=1, \ldots, n$, is a zero-based shift invariant subspace, then the multiplicity of the joint $M_{z}=\left(M_{z_{1}}, \ldots, M_{z_{n}}\right)$-invariant subspace $\left(\mathcal{Q}_{1} \otimes \cdots \otimes \mathcal{Q}_{n}\right)^{\perp}$ of the Dirichlet space or the Hardy space over the unit polydisc in $\mathbb{C}^{n}$ is given by

$$
\operatorname{mult}_{\left.M_{z}\right|_{\left(\mathcal{Q}_{1} \otimes \cdots \otimes \mathcal{Q}_{n}\right)} \perp}\left(\mathcal{Q}_{1} \otimes \cdots \otimes \mathcal{Q}_{n}\right)^{\perp}=\sum_{i=1}^{n}\left(\operatorname{mult}_{\left.M_{z}\right|_{\mathcal{Q}_{i}^{\perp}}}\left(\mathcal{Q}_{i}^{\perp}\right)\right)=n .
$$

A similar result holds for the Bergman space over the unit polydisc.

Keywords: Hardy space; Dirichlet space; Bergman and weighted Bergman spaces; polydisc; rank; multiplicity; joint invariant subspaces; semi-invariant subspaces; zero-based invariant subspaces; tensor product Hilbert spaces

2020 Mathematics subject classifications Primary 47A13; 47A15; 47A16; 47A80; 47B37; 47B38;

Secondary 46C99; 32A35; 32A36; 32A70

\section{Introduction}

This paper is concerned with an additive formula for a numerical invariant of commuting tuples of bounded linear operators on Hilbert spaces. The additive formula arises naturally in connection with a class of simple invariant subspaces of the two-variable Hardy space

* Dedicated to Professor Kalyan Bidhan Sinha on the occasion of his 75th birthday.

(C) The Author(s), 2021. Published by Cambridge University Press on Behalf of The Edinburgh Mathematical Society 
$H^{2}\left(\mathbb{D}^{2}\right)[5]$. From the function Hilbert space point of view, our additive formula is more refined for zero-based invariant subspaces of the Dirichlet space, the Hardy space, the Bergman space and the weighted Bergman spaces over the open unit polydisc $\mathbb{D}^{n}$ in $\mathbb{C}^{n}$.

To be more specific, let us first define the numerical invariant. Given an $n$-tuple of commuting bounded linear operators $T:=\left(T_{1}, \ldots, T_{n}\right)$ on a Hilbert space $\mathcal{H}$, we denote by

$$
\operatorname{mult}_{T}(\mathcal{H})=\min \left\{\# G:[G]_{T}=\mathcal{H}, G \subseteq \mathcal{H}\right\}
$$

where

$$
[G]_{T}=\overline{\operatorname{span}}\left\{T^{k}(G): k \in \mathbb{Z}_{+}^{n}\right\},
$$

and $T^{k}=T_{1}^{k_{1}} \cdots T_{n}^{k_{n}}$ for all $\boldsymbol{k}=\left(k_{1}, \ldots, k_{n}\right) \in \mathbb{Z}_{+}^{n}$. If

$$
\operatorname{mult}_{T}(\mathcal{H})=m<\infty,
$$

then we say that the multiplicity of $T$ is $m$. One also says that $T$ is $m$-cyclic. If $m=1$, then we also say that $T$ is cyclic. A subset $G$ of $\mathcal{H}$ is said to be generating subset with respect to $T$ if $[G]_{T}=\mathcal{H}$.

We pause to note that the computation of multiplicities of (even concrete and simple) bounded linear operators is a challenging problem (perhaps due to its inherent dynamical nature). We refer Rudin [17] for concrete (as well as pathological) examples of invariant subspaces of $H^{2}\left(\mathbb{D}^{2}\right)$ of infinite multiplicities and $[4,5,11-13]$ for some definite results on computations of multiplicities (also see [7]).

The following example, as hinted above, illustrates the complexity of computations of the multiplicities of general function Hilbert spaces. As a first step, we consider the Hardy space $H^{2}(\mathbb{D})$ over $\mathbb{D}$ (the space of all square summable analytic functions on $\mathbb{D}$ ) and the multiplication operator $M_{z}$ by the coordinate function $z$. Let $\mathcal{S}$ be a closed $M_{z}$-invariant subspace of $H^{2}(\mathbb{D})$. Then $\mathcal{Q}=\mathcal{S}^{\perp}$ is a closed $M_{z}^{*}$-invariant subspace of $H^{2}(\mathbb{D})$. It then follows from Beurling that

$$
\operatorname{mult}_{M_{z \mid \mathcal{S}}}(\mathcal{S})=1
$$

that is, $\left.M_{z}\right|_{\mathcal{S}}$ on $\mathcal{S}$ is cyclic. Moreover, taking into account that $\operatorname{mult}_{M_{z}}\left(H^{2}(\mathbb{D})\right)=1$, we obtain (cf. Proposition 2.1)

$$
\operatorname{mult}_{\left.P_{\mathcal{Q}} M_{z}\right|_{\mathcal{Q}}}(\mathcal{Q})=1
$$

where $P_{\mathcal{Q}}$ denotes the orthogonal projection of $H^{2}(\mathbb{D})$ onto $\mathcal{Q}$.

Now we consider the commuting pair of multiplication operators $M_{z}=\left(M_{z_{1}}, M_{z_{2}}\right)$ on $H^{2}\left(\mathbb{D}^{2}\right)$ (the Hardy space over the bidisc). Observe that $H^{2}\left(\mathbb{D}^{2}\right) \cong H^{2}(\mathbb{D}) \otimes H^{2}(\mathbb{D})$. Let $\mathcal{Q}_{1}$ and $\mathcal{Q}_{2}$ be two non-trivial closed $M_{z}^{*}$-invariant subspaces of $H^{2}(\mathbb{D})$. Then $\mathcal{Q}_{1} \otimes \mathcal{Q}_{2}$ is a joint $\left(M_{z_{1}}^{*}, M_{z_{2}}^{*}\right)$-invariant subspace of $H^{2}\left(\mathbb{D}^{2}\right)$, and so $\left(\mathcal{Q}_{1} \otimes \mathcal{Q}_{2}\right)^{\perp}$ is a joint $\left(M_{z_{1}}, M_{z_{2}}\right)$ invariant subspace of $H^{2}\left(\mathbb{D}^{2}\right)$. Set $\left.M_{z}\right|_{\left(\mathcal{Q}_{1} \otimes \mathcal{Q}_{2}\right)^{\perp}}=\left(\left.M_{z_{1}}\right|_{\left(\mathcal{Q}_{1} \otimes \mathcal{Q}_{2}\right)^{\perp}},\left.M_{z_{2}}\right|_{\left.\left(\mathcal{Q}_{1} \otimes \mathcal{Q}_{2}\right)^{\perp}\right)}\right.$. An equivalent reformulation of Douglas and Yang's question (see page 220 in [6] and also [5]) then takes the following form: Is

$$
\operatorname{mult}_{\left.M_{z}\right|_{\left(\mathcal{Q}_{1} \otimes \mathcal{Q}_{2}\right)^{\perp}}}\left(\mathcal{Q}_{1} \otimes \mathcal{Q}_{2}\right)^{\perp}=2 ?
$$

The answer to this question is yes and was obtained by Das along with the first two authors in [5]. This result immediately motivates (see page 1186, [5]) the following natural 
question: Consider the joint $M_{z}=\left(M_{z_{1}}, \ldots, M_{z_{n}}\right)$-invariant subspace $\left(\mathcal{Q}_{1} \otimes \cdots \otimes \mathcal{Q}_{n}\right)^{\perp}$ of $H^{2}\left(\mathbb{D}^{n}\right)$ where $\mathcal{Q}_{1}, \ldots, \mathcal{Q}_{n}$ are non-trivial closed $M_{z}^{*}$-invariant subspaces of $H^{2}(\mathbb{D})$. Is then

$$
\operatorname{mult}_{\left.M_{z}\right|_{\left(\mathcal{Q}_{1} \otimes \cdots \otimes \mathcal{Q}_{n}\right)^{\perp}}}\left(\mathcal{Q}_{1} \otimes \cdots \otimes \mathcal{Q}_{n}\right)^{\perp}=n ?
$$

This can be reformulated more concretely as follows: Let $\mathcal{H}_{i}$ be the Dirichlet space, the Hardy space, the Bergman space, or the weighted Bergman spaces over $\mathbb{D}$ (or, more generally, a reproducing kernel Hilbert spaces of analytic functions on $\mathbb{D}$ for which the operator $M_{z}$ of multiplication by the coordinate function $z$ on $\mathcal{H}_{i}$ is bounded), $i=1, \ldots, n$. Suppose $\mathcal{Q}_{i}^{\perp}$ is an $M_{z}$-invariant closed subspace of $\mathcal{H}_{i}, i=1, \ldots, n$. Is then

$$
\operatorname{mult}_{\left.M_{z} \mid \mathcal{Q}_{1} \otimes \cdots \otimes \mathcal{Q}_{n}\right)^{\perp}}\left(\mathcal{Q}_{1} \otimes \cdots \otimes \mathcal{Q}_{n}\right)^{\perp}=\sum_{i=1}^{n}\left(\operatorname{mult}_{\left.M_{z}\right|_{\mathcal{Q}_{i}^{\perp}}}\left(\mathcal{Q}_{i}^{\perp}\right)\right) ?
$$

In this paper, we aim to propose an approach to verify the above equality for a large class of function Hilbert spaces over $\mathbb{D}^{n}$. The methods and techniques used in this paper are completely different from [5] and can also be applied for proving more powerful results in the setting of general Hilbert spaces. There is indeed a more substantial answer, valid in a larger context of tensor products of Hilbert spaces (see Theorem 4.3).

Let $\mathcal{H} \subseteq \mathcal{O}(\mathbb{D})$ be a reproducing kernel Hilbert space and let the operator $M_{z}$ is bounded on $\mathcal{H}$. Suppose $\mathcal{S}$ is a $M_{z}$-invariant closed subspace of $\mathcal{H}$. We say that $\mathcal{S}$ is a zero-based invariant subspace if there exists $\lambda \in \mathbb{D}$ such that $f(\lambda)=0$ for all $f \in \mathcal{S}$.

A particular case of our main theorem is the following: Let $\mathcal{H}_{i}$ be the Dirichlet space, the Hardy space, the Bergman space, or the weighted Bergman spaces over $\mathbb{D}$. Let $\mathcal{S}_{i}$ be an $M_{z}^{*}$-invariant closed subspace of $\mathcal{H}_{i}, i=1, \ldots, n$. Suppose $\mathcal{S}_{i}:=\mathcal{Q}_{i}^{\perp}$ is a zero-based $M_{z}$-invariant closed subspace of $\mathcal{H}_{i}$ such that

$$
\operatorname{dim}\left(\mathcal{S}_{i} \ominus z \mathcal{S}_{i}\right)<\infty \text { and }\left[\mathcal{S}_{i} \ominus z \mathcal{S}_{i}\right]_{M_{z} \mid \mathcal{S}_{i}}=\mathcal{S}_{i}
$$

for all $i=1, \ldots, n$, then

$$
\operatorname{mult}_{\left.M_{z}\right|_{\left(\mathcal{Q}_{1} \otimes \cdots \otimes \mathcal{Q}_{n}\right)^{\perp}}}\left(\mathcal{Q}_{1} \otimes \cdots \otimes \mathcal{Q}_{n}\right)^{\perp}=\sum_{i=1}^{n}\left(\operatorname{mult}_{\left.M_{z}\right|_{\mathcal{Q}^{\perp}}}\left(\mathcal{Q}_{i}^{\perp}\right)\right)=\sum_{i=1}^{n} \operatorname{dim}\left(\mathcal{S}_{i} \ominus z \mathcal{S}_{i}\right) .
$$

Note that the finite dimensional and generating subspace assumptions are automatically satisfied if $\mathcal{H}_{i}$ is the Hardy space or the Dirichlet space. However, if $\mathcal{S}$ is an $M_{z}$-invariant closed subspace of the Bergman space over $\mathbb{D}$, then

$$
\operatorname{dim}(\mathcal{S} \ominus z \mathcal{S}) \in \mathbb{N} \cup\{\infty\} .
$$

We refer the reader to $[2,8,9]$ for more information. See also [10] for related results in the setting of weighted Bergman spaces over $\mathbb{D}$.

The proof of the above additivity formula uses generating wandering subspace property, geometry of (tensor product) Hilbert spaces, and subspace approximation technique.

The paper is organized as follows. In $\S 2$, we set up notation and prove some basic results on weak multiplicity of (not necessarily commuting) $n$-tuples of operators on Hilbert spaces. In $\S 3$, we study a lower bound multiplicity of joint invariant subspaces 
of a class of commuting $n$-tuples of operators. The main theorem on additivity formula is proved in $\S 4$. The paper is concluded in $\S 5$ with corollaries of the main theorem and some general discussions.

\section{Notation and basic results}

In this section, we introduce the notion of weak multiplicities and describe some preparatory results. This notion is not absolutely needed for the main results of this paper as we shall mostly work in the setting of multiplicities. However, we believe that the idea of weak multiplicities of (not necessary commuting) tuples of operators might be of independent interest. Throughout this paper, the following notation will be adopted: $T_{i}$ is a bounded linear operator on a separable Hilbert space $\mathcal{H}_{i}, i=1, \ldots, n$, and $n \geq 2$. We set

$$
\tilde{\mathcal{H}}=\mathcal{H}_{1} \otimes \cdots \otimes \mathcal{H}_{n}
$$

and

$$
\tilde{T}=\left(\tilde{T}_{1}, \ldots, \tilde{T}_{n}\right)
$$

where

$$
\tilde{T}_{i}=I_{\mathcal{H}_{1}} \otimes \cdots \otimes I_{\mathcal{H}_{i-1}} \otimes T_{i} \otimes I_{\mathcal{H}_{i+1}} \otimes \cdots \otimes I_{\mathcal{H}_{n}} \in \mathcal{B}(\tilde{\mathcal{H}})
$$

for all $i=1, \ldots, n$. It is now clear that $\left(\tilde{T}_{1}, \ldots, \tilde{T}_{n}\right)$ is a doubly commuting tuple of operators on $\tilde{\mathcal{H}}$ (that is, $\tilde{T}_{i} \tilde{T}_{j}=\tilde{T}_{j} \tilde{T}_{i}$ and $\tilde{T}_{p}^{*} \tilde{T}_{q}=\tilde{T}_{q} \tilde{T}_{p}^{*}$ for all $1 \leq i, j \leq n$ and $1 \leq p<$ $q \leq n)$. Moreover, if $\operatorname{mult}_{T_{i}}\left(\mathcal{H}_{i}\right)=1$ for all $i=1, \ldots, n$, then $\operatorname{mult}_{\tilde{T}}(\tilde{\mathcal{H}})=1$. We denote by $\mathbb{D}^{n}$ the unit polydisc in $\mathbb{C}^{n}$ and by $\boldsymbol{z}$ the element $\left(z_{1}, \ldots, z_{n}\right)$ in $\mathbb{C}^{n}$.

The above notion of 'tensor product of operators' is suggested by natural (and analytic) examples of reproducing kernel Hilbert spaces over product domains in $\mathbb{C}^{n}$. For instance, if $\left\{\alpha_{1}, \ldots, \alpha_{n}\right\} \subseteq \mathbb{N}$, then

$$
K_{\boldsymbol{\alpha}}(\boldsymbol{z}, \boldsymbol{w}):=\prod_{i=1}^{n} \frac{1}{\left(1-z_{i} \bar{w}_{i}\right)^{\alpha_{i}}} \quad\left(\boldsymbol{z}, \boldsymbol{w} \in \mathbb{D}^{n}\right)
$$

is a positive definite kernel over $\mathbb{D}^{n}$, and the multiplication operator tuple $\left(M_{z_{1}}, \ldots, M_{z_{n}}\right)$ defines bounded linear operators on the corresponding reproducing kernel Hilbert space $L_{\boldsymbol{\alpha}}^{2}\left(\mathbb{D}^{n}\right)$ (known as the weighted Bergman space over $\mathbb{D}^{n}$ with weight $\boldsymbol{\alpha}=\left(\alpha_{1}, \ldots, \alpha_{n}\right)$ ). It follows that (cf. [19])

$$
\tilde{\mathcal{H}}=L_{\alpha_{1}}^{2}(\mathbb{D}) \otimes \cdots \otimes L_{\alpha_{n}}^{2}(\mathbb{D}), \quad \text { and } \quad \tilde{M}_{z}=\left(\tilde{M}_{z_{1}}, \ldots, \tilde{M}_{z_{n}}\right),
$$

where $M_{z_{i}}$ denotes the multiplication operator $M_{z}$ on $L_{\alpha_{i}}^{2}(\mathbb{D}), i=1, \ldots, n$. In particular, if $\boldsymbol{\alpha}=(1, \ldots, 1)$, then $\tilde{\mathcal{H}}=H^{2}\left(\mathbb{D}^{n}\right)$ is the well-known Hardy space over the unit polydisc. We also refer the reader to Popescu $[14,15]$ for elegant and rich theory of 'tensor product of operators' in multivariable operator theory. 
Let $\mathcal{H}$ be a Hilbert space, and let $A=\left(A_{1}, \ldots, A_{n}\right)$ be an $n$-tuple (not necessarily commuting) of bounded linear operators on $\mathcal{H}$. Let

$$
\mathrm{w}-m u l t_{A}(\mathcal{H})=\min \left\{\# G:[G]_{A}=\mathcal{H}, G \subseteq \mathcal{H}\right\},
$$

where

$$
[G]_{A}=\overline{\operatorname{span}}\left\{A^{k}(G): k \in \mathbb{Z}_{+}^{n}\right\},
$$

and $A^{k}=A_{1}^{k_{1}} \cdots A_{n}^{k_{n}}$ for all $k \in \mathbb{Z}_{+}^{n}$. If w-mult ${ }_{A}(\mathcal{H})=m<\infty$, then we say that the weak multiplicity of $A$ is $m$. We say that $A$ is weakly cyclic if w-mult ${ }_{A}(\mathcal{H})=1$. A subset $G$ of $\mathcal{H}$ is said to be weakly generating with respect to $A$ if $[G]_{A}=\mathcal{H}$.

Now let $\mathcal{L}$ be a closed subspace of $\mathcal{H}$. Then

$$
\mathcal{W}_{A}(\mathcal{L}):=\mathcal{L} \ominus \sum_{i=1}^{n} A_{i} \mathcal{L},
$$

is called the wandering subspace of $\mathcal{L}$ with respect to $\left.P_{\mathcal{L}} A\right|_{\mathcal{L}}$. If, in addition

$$
\mathcal{L}=\bigvee_{k \in \mathbb{Z}_{+}^{n}}\left(\left.P_{\mathcal{L}} A\right|_{\mathcal{L}}\right)^{k}\left(\mathcal{W}_{A}(\mathcal{L})\right)
$$

then we say that $\left.P_{\mathcal{L}} A\right|_{\mathcal{L}}$ satisfies the weakly generating wandering subspace property. Here $\left.P_{\mathcal{L}} A\right|_{\mathcal{L}}=\left(\left.P_{\mathcal{L}} A_{1}\right|_{\mathcal{L}}, \ldots,\left.P_{\mathcal{L}} A_{n}\right|_{\mathcal{L}}\right)$ and

$$
\left(\left.P_{\mathcal{L}} A\right|_{\mathcal{L}}\right)^{k}=\left(\left.P_{\mathcal{L}} A_{1}\right|_{\mathcal{L}}\right)^{k_{1}} \cdots\left(\left.P_{\mathcal{L}} A_{n}\right|_{\mathcal{L}}\right)^{k_{n}},
$$

for all $k \in \mathbb{Z}_{+}^{n}$.

Note that if $A$ is commuting and $\mathcal{L}$ is joint $A$-invariant subspace (that is, $A_{i} \mathcal{L} \subseteq \mathcal{L}$ for all $i=1 \ldots, n)$, then weakly generating wandering subspace property is commonly known as generating wandering subspace property.

We now proceed to relate weak multiplicities and dimensions of weakly generating wandering subspaces. Let $A$ be an $n$-tuple of bounded linear operators on $\mathcal{H}, \mathcal{L}$ be a joint $A$-invariant subspace of $\mathcal{H}$, and let $\mathcal{M}$ be a closed subspace of $\mathcal{L}$. Then

$$
P_{\mathcal{W}_{A}(\mathcal{L})}\left([\mathcal{M}]_{A}\right)=P_{\mathcal{W}_{A}(\mathcal{L})}(\mathcal{M})
$$

since

$$
P_{\mathcal{W}_{A}(\mathcal{L})}\left(A^{k} \mathcal{M}\right)=0 \text { for all } k \in \mathbb{Z}_{+}^{n} \backslash\{0\}
$$

Now suppose that $[\mathcal{M}]_{A}=\mathcal{L}$, that is, $\mathcal{M}$ is a weakly generating subspace of $\mathcal{L}$ with respect to $A$. Then

$$
\mathcal{W}_{A}(\mathcal{L})=P_{\mathcal{W}_{A}(\mathcal{L})}(\mathcal{M})
$$

Hence

$$
\mathrm{w}_{-} \operatorname{mult}_{\left.A\right|_{\mathcal{L}}}(\mathcal{L}) \geq \operatorname{dim} \mathcal{W}_{A}(\mathcal{L})
$$

Moreover, if $\mathcal{L}$ satisfies the weakly generating wandering subspace property, then

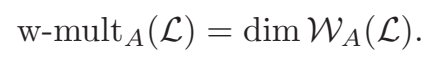

Therefore, we have proved the following: 
Proposition 2.1. Let $\mathcal{L}$ be a closed joint $A$-invariant subspace of $\mathcal{H}$. If $\mathcal{L}$ satisfies the weakly generating wandering subspace property with respect to $A_{\mathcal{L}}$, then $\mathrm{w}_{\text {-mult }}(\mathcal{L})=$ $\operatorname{dim} \mathcal{W}_{A}(\mathcal{L})$.

We now proceed to a variation of Lemma 2.1 in [5] which relates the multiplicity of a commuting tuple of operators with the weak-multiplicity of the compressed tuple to a semi-invariant subspace.

Lemma 2.2. Let $A$ be an $n$-tuple of bounded linear operators on a Hilbert space $\mathcal{H}$. Let $\mathcal{L}_{1}$ and $\mathcal{L}_{2}$ be two joint $A$-invariant subspaces of $\mathcal{H}$ and $\mathcal{L}_{2} \subseteq \mathcal{L}_{1}$. If $\mathcal{L}=\mathcal{L}_{1} \ominus \mathcal{L}_{2}$, then

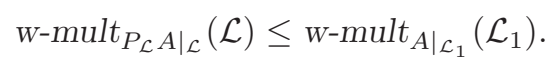

Proof. We have $P_{\mathcal{L}} A_{j} P_{\mathcal{L}}=P_{\mathcal{L}} A_{j} P_{\mathcal{L}_{1}}-P_{\mathcal{L}} A_{j} P_{\mathcal{L}_{2}}$ and thus by $A_{j} \mathcal{L}_{2} \subseteq \mathcal{L}_{2}$ we infer that

$$
P_{\mathcal{L}} A_{j} P_{\mathcal{L}}=P_{\mathcal{L}} A_{j} P_{\mathcal{L}_{1}}
$$

for all $j=1, \ldots, n$. Since $A_{j} \mathcal{L}_{1} \subseteq \mathcal{L}_{1}$, we have

$$
\left(P_{\mathcal{L}} A_{i} P_{\mathcal{L}}\right)\left(P_{\mathcal{L}} A_{j} P_{\mathcal{L}}\right)=P_{\mathcal{L}} A_{i} P_{\mathcal{L}_{1}} A_{j} P_{\mathcal{L}_{1}}
$$

that is

$$
\left(P_{\mathcal{L}} A_{i} P_{\mathcal{L}}\right)\left(P_{\mathcal{L}} A_{j} P_{\mathcal{L}}\right)=P_{\mathcal{L}}\left(A_{i} A_{j}\right) P_{\mathcal{L}_{1}},
$$

for all $i, j=1, \ldots, n$, and so

$$
\left(P_{\mathcal{L}} A P_{\mathcal{L}}\right)^{k}=P_{\mathcal{L}} A^{k} P_{\mathcal{L}_{1}}
$$

for all $\boldsymbol{k} \in \mathbb{Z}_{+}^{n}$. Clearly, if $G$ is a minimal generating subset of $\mathcal{L}_{1}$ with respect to $\left.A\right|_{\mathcal{L}_{1}}$, then $P_{\mathcal{L}} G$ is a generating subset of $\mathcal{L}$ with respect to $\left.P_{\mathcal{L}} A\right|_{\mathcal{L}}$, and thus w-mult $\left.P_{\mathcal{L}} A\right|_{\mathcal{L}}(\mathcal{L}) \leq$ w-mult ${ }_{A \mid \mathcal{L}_{1}}\left(\mathcal{L}_{1}\right)$. This completes the proof of the lemma.

In particular, if $\mathcal{L}_{1}=\mathcal{H}$, then $\mathcal{Q}:=\mathcal{H} \ominus \mathcal{L}_{2}$ is a joint $\left(A_{1}^{*}, \ldots, A_{n}^{*}\right)$-invariant subspace of $\mathcal{H}$. In this case, denote by $C_{i}=\left.P_{\mathcal{Q}} A_{i}\right|_{\mathcal{Q}}$ the compression of $A_{i}, i=1, \ldots, n$, and define the $n$-tuple on $\mathcal{Q}$ as

$$
C_{\mathcal{Q}}=\left(C_{1}, \ldots, C_{n}\right)
$$

Then we have the following estimate:

$$
{\mathrm{w}-\operatorname{mult}_{C_{\mathcal{Q}}}(\mathcal{Q}) \leq \mathrm{w}^{-m_{u l t}}{ }_{A}(\mathcal{H})}
$$

Moreover, we also have

Corollary 2.3. Let $A=\left(A_{1}, \ldots, A_{n}\right)$ be a commuting tuple of bounded linear operators on a Hilbert space $\mathcal{H}$. If $\mathcal{Q}$ is a closed joint $A^{*}$-invariant subspace of $\mathcal{H}$, then

$$
\operatorname{mult}_{C_{\mathcal{Q}}}(\mathcal{Q}) \leq \operatorname{mult}_{A}(\mathcal{H}) .
$$

This has the following immediate (and well-known) application: Suppose $A$ is a commuting tuple on $\mathcal{H}$. If $A$ is cyclic, then $C_{\mathcal{Q}}$ on $\mathcal{Q}$ is also cyclic. 


\section{A lower bound for multiplicities}

In this section, we first lay out the setting of joint invariant subspaces of our discussions throughout the paper. Then we present a lower bound of multiplicities of those joint invariant subspaces. We begin by recalling the following useful lemma (cf. Lemma 2.5, [18]):

Lemma 3.1. If $\left\{A_{i}\right\}_{i=1}^{n}$ is a commuting set of orthogonal projections on a Hilbert space $\mathcal{K}$, then $\mathcal{L}=\sum_{i=1}^{n} \operatorname{ran}_{i}$ is a closed subspace of $\mathcal{K}$, and

$$
\begin{aligned}
P_{\mathcal{L}} & =I-\prod_{i=1}^{n}\left(I-A_{i}\right) \\
& =A_{1}\left(I-A_{2}\right) \ldots\left(I-A_{n}\right) \oplus A_{2}\left(I-A_{3}\right) \ldots\left(I-A_{n}\right) \oplus \ldots+A_{n-1}\left(I-A_{n}\right) \oplus A_{n} .
\end{aligned}
$$

Next, we introduce the invariant subspaces of interest. Again, we continue to follow the notation as introduced in $\S 2$.

Let $\mathcal{H}_{i}$ be a Hilbert space, $T_{i}$ a bounded linear operator on $\mathcal{H}_{i}$, and let $\mathcal{Q}_{i}$ be a closed $T_{i}^{*}$-invariant subspace of $\mathcal{H}_{i}, i=1, \ldots, n$. Set $\mathcal{S}_{i}=\mathcal{Q}_{i}^{\perp}$ and

$$
P_{i}=P_{\mathcal{S}_{i}} \quad \text { and } \quad Q_{i}=I_{\mathcal{H}_{i}}-P_{\mathcal{S}_{i}},
$$

for all $i=1, \ldots, n$. Recall again that

$$
\tilde{P}_{i}=I_{\mathcal{H}_{1}} \otimes \ldots \otimes I_{\mathcal{H}_{i-1}} \otimes P_{\mathcal{S}_{i}} \otimes I_{\mathcal{H}_{i+1}} \otimes \ldots \otimes I_{\mathcal{H}_{n}} \in \mathcal{B}(\tilde{\mathcal{H}})
$$

and

$$
\tilde{P}_{i} \tilde{P}_{j}=\tilde{P}_{j} \tilde{P}_{i}
$$

for all $i, j=1, \ldots, n$. By Lemma 3.1, it then follows that

$$
\mathcal{S}=\sum_{i=1}^{n} \operatorname{ran} \tilde{P}_{i}
$$

is a joint $\tilde{T}$-invariant subspace of $\tilde{\mathcal{H}}$. Moreover,

$$
\mathcal{S}=\left(\mathcal{Q}_{1} \otimes \cdots \otimes \mathcal{Q}_{n}\right)^{\perp}
$$

Our main goal is to compute the multiplicity of the commuting tuple $\left.\tilde{T}\right|_{\mathcal{S}}=$ $\left(\left.\tilde{T}_{1}\right|_{\mathcal{S}}, \ldots,\left.\tilde{T}_{n}\right|_{\mathcal{S}}\right)$ on $\mathcal{S}$. 
For each $i=1, \ldots, n$, define $X_{i} \in \mathcal{B}(\tilde{\mathcal{H}})$ by

$$
X_{i}=\tilde{P}_{i} \tilde{Q}_{i+1} \ldots \tilde{Q}_{n} .
$$

Then $X_{i}^{2}=X_{i}=X_{i}^{*}$ and

$$
X_{p} X_{q}=0
$$

for all $i=1, \ldots, n$, and $p \neq q$. This implies that $\left\{X_{i}\right\}_{i=1}^{n}$ is a set of orthogonal projections with orthogonal ranges. Then, by virtue of (3.1), one can further rewrite $\mathcal{S}$ as

$$
\mathcal{S}=\sum_{i=1}^{n} \operatorname{ran} \tilde{P}_{i}=\bigoplus_{i=1}^{n} \operatorname{ran} X_{i},
$$

and by Lemma 3.1, one represents $P_{\mathcal{S}}$ as

$$
P_{\mathcal{S}}=\bigoplus_{i=1}^{n} X_{i} .
$$

Define

$$
\mathcal{F}=\operatorname{ran} X_{1} \oplus \operatorname{ran}\left(\tilde{Q}_{1} X_{2}\right) \oplus \cdots \oplus \operatorname{ran}\left(\tilde{Q}_{1} \cdots \tilde{Q}_{n-1} X_{n}\right) .
$$

Then, as easily seen

$$
\tilde{Q}_{i} X_{j}=X_{j} \tilde{Q}_{i}
$$

for all $1 \leq i \leq j$ and $j=1, \ldots, n$, it follows that

$$
\operatorname{ran}\left(\tilde{Q}_{1} \cdots \tilde{Q}_{p} X_{p+1}\right) \subseteq \operatorname{ran} X_{p+1},
$$

for all $p=1, \ldots, n-1$, and consequently

$$
\mathcal{S} \supseteq \mathcal{F} .
$$

Our first aim is to analyse the closed subspace $\mathcal{F}$ and to construct $n-1$ nested (and suitable) closed subspaces $\left\{\mathcal{F}_{i}\right\}_{i=1}^{n-1}$ such that

$$
\mathcal{S} \supseteq \mathcal{F}_{1} \supseteq \cdots \supseteq \mathcal{F}_{n-1}=\mathcal{F} \text {. }
$$

To this end, first set

$$
\mathcal{F}_{1}=\operatorname{ran} X_{1} \oplus \operatorname{ran} X_{2} \oplus \cdots \oplus \operatorname{ran} X_{n-1} \oplus \operatorname{ran}\left(\tilde{Q}_{n-1} X_{n}\right),
$$

and define

$$
\mathcal{F}_{2}=\operatorname{ran} X_{1} \oplus \operatorname{ran}\left(\tilde{Q}_{1} X_{2}\right) \oplus \cdots \oplus \operatorname{ran}\left(\tilde{Q}_{1} X_{n-1}\right) \oplus \operatorname{ran}\left(\tilde{Q}_{1} \tilde{Q}_{n-1} X_{n}\right) .
$$

We then proceed to define $\mathcal{F}_{i}, i=2, \ldots, n-1$, as

$$
\begin{aligned}
\mathcal{F}_{i}= & \operatorname{ran}\left(X_{1} \oplus \tilde{Q}_{1} X_{2} \oplus \cdots \oplus\left(\prod_{t=1}^{i-1} \tilde{Q}_{t}\right) X_{i} \oplus \cdots \oplus\left(\prod_{t=1}^{i-1} \tilde{Q}_{t}\right) X_{n-1}\right. \\
& \left.\oplus\left(\prod_{t=1}^{i-1} \tilde{Q}_{t} \tilde{Q}_{n-1}\right) X_{n}\right) .
\end{aligned}
$$


Therefore

$$
P_{\mathcal{F}_{i}}=X_{1} \oplus \tilde{Q}_{1} X_{2} \oplus \cdots \oplus\left(\prod_{t=1}^{i-1} \tilde{Q}_{t}\right) X_{i} \oplus \cdots \oplus\left(\prod_{t=1}^{i-1} \tilde{Q}_{t}\right) X_{n-1} \oplus\left(\prod_{t=1}^{i-1} \tilde{Q}_{t} \tilde{Q}_{n-1}\right) X_{n}
$$

for all $i=2, \ldots, n-1$. Therefore, denoting

$$
A=\left(\prod_{t=1}^{i-2} \tilde{Q}_{t}\right) \tilde{P}_{i-1}
$$

we have

$$
P_{\mathcal{F}_{i-1} \ominus \mathcal{F}_{i}}=A\left(X_{i} \oplus X_{i+1} \oplus \cdots \oplus X_{n-1} \oplus \tilde{Q}_{n-1} X_{n}\right),
$$

for all $i=2, \ldots, n-1$. Since $A X_{p}=X_{p} A$ for all $p=i, \ldots, n$, the above formula yields

$$
P_{\mathcal{F}_{i-1} \ominus \mathcal{F}_{i}}=\left(X_{i} \oplus X_{i+1} \oplus \cdots \oplus X_{n-1} \oplus \tilde{Q}_{n-1} X_{n}\right) A .
$$

Let $i \in\{2, \ldots, n-1\}$ be a fixed natural number. We claim that $\mathcal{F}_{i-1} \ominus \mathcal{F}_{i}$ is a joint $P_{\mathcal{F}_{i-1}} \tilde{T} P_{\mathcal{F}_{i-1}}$-invariant subspace, that is

$$
P_{\mathcal{F}_{i-1}} \tilde{T}_{j}\left(\mathcal{F}_{i-1} \ominus \mathcal{F}_{i}\right) \subseteq \mathcal{F}_{i-1} \ominus \mathcal{F}_{i}
$$

or, equivalently

$$
\left(P_{\mathcal{F}_{i-1}} \tilde{T}_{j} P_{\mathcal{F}_{i-1}}\right) P_{\mathcal{F}_{i-1} \ominus \mathcal{F}_{i}}=\left.P_{\mathcal{F}_{i-1} \ominus \mathcal{F}_{i}} \tilde{T}_{j}\right|_{\mathcal{F}_{i-1} \ominus \mathcal{F}_{i}},
$$

for all $j=1, \ldots, n$. There are four cases:

Case I: If $j>i$, then one has $\tilde{T}_{j} A=A \tilde{T}_{j}$ and so

$$
P_{\mathcal{F}_{i-1} \ominus \mathcal{F}_{i}} \tilde{T}_{j} P_{\mathcal{F}_{i-1} \ominus \mathcal{F}_{i}}=A\left(X_{i} \oplus X_{i+1} \oplus \cdots \oplus \tilde{Q}_{n-1} X_{n}\right) \tilde{T}_{j}\left(X_{i} \oplus X_{i+1} \oplus \cdots \oplus \tilde{Q}_{n-1} X_{n}\right) .
$$

On the other hand, since

$$
P_{\mathcal{F}_{i-1}} \tilde{T}_{j} P_{\mathcal{F}_{i-1} \ominus \mathcal{F}_{i}}=P_{\mathcal{F}_{i-1}} A \tilde{T}_{j}\left(X_{i} \oplus \cdots \oplus X_{j} \oplus \cdots \oplus \tilde{Q}_{n-1} X_{n}\right),
$$

and

$$
\begin{aligned}
P_{\mathcal{F}_{i-1}}= & X_{1} \oplus\left(\tilde{Q}_{1} X_{2}\right) \oplus \cdots \oplus\left(\prod_{t=1}^{i-2} \tilde{Q}_{t} X_{i-1}\right) \oplus\left(\prod_{t=1}^{i-2} \tilde{Q}_{t} X_{i}\right) \\
& \oplus \cdots \oplus\left(\prod_{t=1}^{i-2} \tilde{Q}_{t} X_{n-1}\right) \oplus\left(\prod_{t=1}^{i-2} \tilde{Q}_{t} \tilde{Q}_{n-1} X_{n}\right)
\end{aligned}
$$

it follows that

$$
P_{\mathcal{F}_{i-1}} \tilde{T}_{j} P_{\mathcal{F}_{i-1} \ominus \mathcal{F}_{i}}=A\left(X_{i-1} \oplus X_{i} \oplus \cdots \oplus \tilde{Q}_{n-1} X_{n}\right) \tilde{T}_{j}\left(X_{i} \oplus \cdots \oplus \tilde{Q}_{n-1} X_{n}\right),
$$

as $X_{t} A=0$ for all $t=1, \ldots, i-2$, and $\prod_{t=1}^{i-2} \tilde{Q}_{t} A=A$. Moreover, since

$$
X_{i-1} \tilde{T}_{j}=\left(\tilde{P}_{i-1} \tilde{Q}_{i} \cdots \tilde{Q}_{j} \cdots \tilde{Q}_{n}\right) \tilde{T}_{j}=\tilde{P}_{i-1} \tilde{Q}_{i} \cdots \widetilde{Q_{j} T_{j} Q_{j}} \cdots \tilde{Q}_{n},
$$


it follows that

$$
X_{i-1} \tilde{T}_{j} X_{t}=0
$$

for all $t=i, \ldots, n$. This leads to

$$
P_{\mathcal{F}_{i-1}} \tilde{T}_{j} P_{\mathcal{F}_{i-1} \ominus \mathcal{F}_{i}}=A\left(X_{i} \oplus X_{i+1} \oplus \cdots \oplus \tilde{Q}_{n-1} X_{n}\right) \tilde{T}_{j}\left(X_{i} \oplus X_{i+1} \oplus \cdots \oplus \tilde{Q}_{n-1} X_{n}\right) .
$$

Case II: If $j=i$, then

$$
\tilde{T}_{i} P_{\mathcal{F}_{i-1} \ominus \mathcal{F}_{i}}=A\left(\left(\widetilde{T_{i} P_{i}} \tilde{Q}_{i+1} \cdots \tilde{Q}_{n}\right) \oplus \tilde{T}_{i} X_{i+1} \oplus \cdots \oplus \tilde{T}_{i} \tilde{Q}_{n-1} X_{n}\right),
$$

implies that

$$
\begin{aligned}
P_{\mathcal{F}_{i-1}} \tilde{T}_{i} P_{\mathcal{F}_{i-1} \ominus \mathcal{F}_{i}}= & \left(\prod_{t=1}^{i-2} \tilde{Q}_{t}\right)\left(X_{i-1} \oplus X_{i} \oplus \cdots \oplus \tilde{Q}_{n-1} X_{n}\right) \tilde{T}_{i} P_{\mathcal{F}_{i-1} \ominus \mathcal{F}_{i}} \\
= & \left(\prod_{t=1}^{i-2} \tilde{Q}_{t}\right)\left(X_{i-1} \oplus X_{i} \oplus \cdots \oplus \tilde{Q}_{n-1} X_{n}\right) \tilde{T}_{i} A \\
& \times\left(X_{i} \oplus X_{i+1} \oplus \cdots \oplus \tilde{Q}_{n-1} X_{n}\right) \\
= & A\left(X_{i-1} \oplus X_{i} \oplus \cdots \oplus \tilde{Q}_{n-1} X_{n}\right) \tilde{T}_{i}\left(X_{i} \oplus X_{i+1} \oplus \cdots \oplus \tilde{Q}_{n-1} X_{n}\right) \\
= & P_{\mathcal{F}_{i-1} \ominus \mathcal{F}_{i}} \tilde{T}_{i} P_{\mathcal{F}_{i-1} \ominus \mathcal{F}_{i}},
\end{aligned}
$$

where the next-to-last equality follows from the fact again that $A \tilde{T}_{i}=\tilde{T}_{i} A,\left(\prod_{t=1}^{i-2} \tilde{Q}_{t}\right) A=$ $A$ and $X_{i-1} \tilde{T}_{i} X_{t}=0$ for all $t=i, \ldots, n$.

Case III: Let $j=i-1$. Since

$$
\tilde{T}_{i-1} A=\left(\prod_{t=1}^{i-2} \tilde{Q}_{t}\right) \widetilde{T_{i-1} P_{i-1}}=\widehat{A T_{i-1} P_{i-1}},
$$

by setting

$$
\hat{A}=\left(\prod_{t=1}^{i-2} \tilde{Q}_{t}\right) \widetilde{T_{i-1} P_{i-1}},
$$

it follows that

$$
\tilde{T}_{i-1} P_{\mathcal{F}_{i-1} \ominus \mathcal{F}_{i}}=\hat{A} X_{i} \oplus \hat{A} X_{i+1} \oplus \cdots \oplus \hat{A} X_{n-1} \oplus \hat{A} \tilde{Q}_{n-1} X_{n}
$$

Then $X_{p} \hat{A}=\hat{A} X_{p}$ for all $p=i, \ldots, n$, and $A \hat{A}=\hat{A}$ implies that

$$
\begin{aligned}
P_{\mathcal{F}_{i-1}} \tilde{T}_{i-1} P_{\mathcal{F}_{i-1} \ominus \mathcal{F}_{i}} & =\hat{A}\left(X_{i} \oplus X_{i+1} \oplus \cdots \oplus X_{n-1} \oplus \tilde{Q}_{n-1} X_{n}\right) \\
& =P_{\mathcal{F}_{i-1} \ominus \mathcal{F}_{i}} \tilde{T}_{i-1} P_{\mathcal{F}_{i-1} \ominus \mathcal{F}_{i}}
\end{aligned}
$$

where the second equality follows from (3.5) and the fact that $T_{i-1} P_{i-1}=P_{i-1} T_{i-1} P_{i-1}$. 
Case IV: Let $j<i-1$. Then it is clear that

$$
\tilde{T}_{j} P_{\mathcal{F}_{i-1} \ominus \mathcal{F}_{i}}=\hat{A}\left(X_{i} \oplus X_{i+1} \oplus \cdots \oplus X_{n-1} \oplus \tilde{Q}_{n-1} X_{n}\right),
$$

where $\hat{A}=\tilde{T}_{j} A$, that is

$$
\hat{A}=\tilde{Q}_{1} \cdots \tilde{Q}_{j-1} \widetilde{T_{j} Q_{j}} \tilde{Q}_{j+1} \cdots \tilde{Q}_{i-2} \tilde{P}_{i-1}
$$

Note that $X_{t} \hat{A}=\hat{A} X_{t}$ for all $t=i, \ldots, n$, and

$$
A \hat{A}=\tilde{Q}_{1} \cdots \tilde{Q}_{j-1}{\widetilde{Q_{j} T_{j} Q_{j}}}_{Q_{j+1}} \cdots \tilde{Q}_{i-2} \tilde{P}_{i-1}
$$

Since $X_{p} X_{q}=\delta_{p q} X_{p}$ for all $p$ and $q$, it follows that

$$
P_{\mathcal{F}_{i-1}} \tilde{T}_{j} P_{\mathcal{F}_{i-1} \ominus \mathcal{F}_{i}}=A \hat{A}\left(X_{i} \oplus X_{i+1} \oplus \cdots \oplus X_{n-1} \oplus \tilde{Q}_{n-1} X_{n}\right)
$$

On the other hand, the representation of $\tilde{T}_{j} P_{\mathcal{F}_{i-1} \ominus \mathcal{F}_{i}}$ above and (3.5) yields

$$
P_{\mathcal{F}_{i-1} \ominus \mathcal{F}_{i}} \tilde{T}_{j} P_{\mathcal{F}_{i-1} \ominus \mathcal{F}_{i}}=A \hat{A}\left(X_{i} \oplus X_{i+1} \oplus \cdots \oplus X_{n-1} \oplus \tilde{Q}_{n-1} X_{n}\right)
$$

and proves the claim.

We turn now to prove that $\left(P_{\mathcal{F}_{i}} \tilde{T}_{1}\left|\mathcal{F}_{i}, \ldots, P_{\mathcal{F}_{i}} \tilde{T}_{n}\right| \mathcal{F}_{i}\right)$ is a commuting tuple for all $i=1, \ldots, n-1$, that is

$$
P_{\mathcal{F}_{i}} \tilde{T}_{s} P_{\mathcal{F}_{i}} \tilde{T}_{t} P_{\mathcal{F}_{i}}=P_{\mathcal{F}_{i}} \tilde{T}_{t} P_{\mathcal{F}_{i}} \tilde{T}_{s} P_{\mathcal{F}_{i}}
$$

for all $s, t=1, \ldots, n$. Fix an $i \in\{1, \ldots, n-1\}$ and let

$$
P_{\mathcal{F}_{i}}=M_{1} \oplus \cdots \oplus M_{n}
$$

where $M_{j}, j=1, \ldots, n$, denotes the $j$ th summand in the representation of $P_{\mathcal{F}_{i}}$ in (3.4). Recalling the terms in (3.4), we see that $M_{j}$ is a product of $n$ distinct commuting orthogonal projections of the form $\tilde{P}_{k}, \tilde{Q}_{l}$ and $\tilde{I}_{\mathcal{H}_{m}}, 1 \leq k, l, m \leq n$. For each $s=1, \ldots, n$, we set

$$
M_{j}=M_{j, s} \hat{M}_{j, s}
$$

where $M_{j, s}$ is the $s$ th factor of $M_{j}$ and $\hat{M}_{j, s}$ is the product of the same factors of $M_{j}$, except the $s$ th factor of $M_{j}$ is replaced by $\tilde{I}_{\mathcal{H}_{s}}$. Note again that $M_{j, s}=\tilde{P}_{s}, \tilde{Q}_{s}$, or $\tilde{I}_{\mathcal{H}_{s}}$. 
We first claim that

$$
M_{j} \tilde{T}_{s} M_{k}=0
$$

for all $j \neq k$. Indeed, if $M_{j, s}=\tilde{Q}_{s}$, then $M_{j} \tilde{T}_{s} M_{k}=M_{j, s} \hat{M}_{j, s} \tilde{T}_{s} M_{k}$ yields

$$
M_{j} \tilde{T}_{s} M_{k}=M_{j, s} \tilde{T}_{s} \hat{M}_{j, s} M_{k}=M_{j, s} \tilde{T}_{s} M_{j, s} \hat{M}_{j, s} M_{k}=M_{j, s} \tilde{T}_{s} M_{j} M_{k}=0,
$$

as $\tilde{Q}_{s} \tilde{T}_{s} \tilde{Q}_{s}=\tilde{Q}_{s} \tilde{T}_{s}$. Similarly, if $M_{j, s}=\tilde{P}_{s}$, then

$$
M_{j} \tilde{T}_{s} M_{k}=M_{j} \hat{M}_{k, s} \tilde{T}_{s} M_{k, s}=M_{j} \hat{M}_{k, s} M_{k, s} \tilde{T}_{s} M_{k, s}=M_{j} M_{k} \tilde{T}_{s} M_{k, s}=0,
$$

as $\tilde{P}_{s} \tilde{T}_{s} \tilde{P}_{s}=\tilde{T}_{s} \tilde{P}_{s}$. The remaining case, $M_{j, s}=\tilde{I}_{\mathcal{H}_{s}}$, follows from the fact that

$$
M_{j} \tilde{T}_{s} M_{k}=\tilde{T}_{s} M_{j} M_{k}
$$

This proves the claim. Hence the representation of $P_{\mathcal{F}_{i}} \tilde{T}_{s} P_{\mathcal{F}_{i}}$ simplifies as

$$
P_{\mathcal{F}_{i}} \tilde{T}_{s} P_{\mathcal{F}_{i}}=M_{1} \tilde{T}_{s} M_{1} \oplus \cdots \oplus M_{n} \tilde{T}_{s} M_{n}
$$

Thus,

$$
P_{\mathcal{F}_{i}} \tilde{T}_{s} P_{\mathcal{F}_{i}} \tilde{T}_{t} P_{\mathcal{F}_{i}}=M_{1} \tilde{T}_{s} M_{1} \tilde{T}_{t} M_{1} \oplus \cdots \oplus M_{n} \tilde{T}_{s} M_{n} \tilde{T}_{t} M_{n}
$$

Now if $s \neq t$, then for each $j=1, \ldots, n$, we have

$$
\begin{aligned}
M_{j} \tilde{T}_{s} M_{j} \tilde{T}_{t} M_{j} & =M_{j} \hat{M}_{j, s} \tilde{T}_{s} M_{j, s} M_{j, t} \tilde{T}_{t} \hat{M}_{j, t} M_{j} \\
& =\left(M_{j} \hat{M}_{j, s} M_{j, t}\right) \tilde{T}_{s} \tilde{T}_{t}\left(M_{j, s} \hat{M}_{j, t} M_{j}\right) \\
& =M_{j} \tilde{T}_{s} \tilde{T}_{t} M_{j}
\end{aligned}
$$

and hence

$$
\left(P_{\mathcal{F}_{i}} \tilde{T}_{s} P_{\mathcal{F}_{i}}\right)\left(P_{\mathcal{F}_{i}} \tilde{T}_{t} P_{\mathcal{F}_{i}}\right)=M_{1} \tilde{T}_{s} \tilde{T}_{t} M_{1} \oplus \cdots \oplus M_{n} \tilde{T}_{s} \tilde{T}_{t} M_{n}
$$

This completes the proof of the commutativity property of the tuple $\left(\left.P_{\mathcal{F}_{i}} \tilde{T}_{1}\right|_{\mathcal{F}_{i}}, \ldots\right.$, $\left.\left.P_{\mathcal{F}_{i}} \tilde{T}_{n}\right|_{\mathcal{F}_{i}}\right), i=1, \ldots, n-1$. Furthermore, if $s=t$, then

$$
\left(M_{j} \tilde{T}_{s} M_{j}\right)^{2}=M_{j} \tilde{T}_{s}^{2} M_{j}
$$

Indeed, if $M_{j, s}=\tilde{Q}_{s}$, then $M_{j} \tilde{T}_{s} M_{j}=M_{j} \tilde{T}_{s} \hat{M}_{j, s}$ gives us

$$
M_{j} \tilde{T}_{s} M_{j} \tilde{T}_{s} M_{j}=M_{j} \tilde{T}_{s} \hat{M}_{j, s} \tilde{T}_{s} \hat{M}_{j, s} M_{j}=M_{j} \tilde{T}_{s} \tilde{T}_{s} \hat{M}_{j, s} M_{j}=M_{j} \tilde{T}_{s}^{2} M_{j}
$$

Similarly, if $M_{j, s}=\tilde{P}_{s}$ or $\tilde{I}_{\mathcal{H}_{s}}$, then $M_{j} \tilde{T}_{s} M_{j}=\tilde{T}_{s} M_{j}$, and hence

$$
M_{j} \tilde{T}_{s} M_{j} \tilde{T}_{s} M_{j}=M_{j} \tilde{T}_{s}^{2} M_{j}
$$

Hence we obtain

$$
\left(P_{\mathcal{F}_{i}} \tilde{T}_{s} P_{\mathcal{F}_{i}}\right)\left(P_{\mathcal{F}_{i}} \tilde{T}_{t} P_{\mathcal{F}_{i}}\right)=M_{1} \tilde{T}_{s} \tilde{T}_{t} M_{1} \oplus \cdots \oplus M_{n} \tilde{T}_{s} \tilde{T}_{t} M_{n}
$$

for all $s, t=1, \ldots, n$.

Therefore, with the notation introduced above, we have proved the following: 
Lemma 3.2. If $\mathcal{S}=\left(\mathcal{Q}_{1} \otimes \cdots \otimes \mathcal{Q}_{n}\right)^{\perp}$, then $\mathcal{S}$ is a joint $\tilde{T}$-invariant subspace of $\tilde{\mathcal{H}}$ and

$$
\mathcal{S} \supseteq \mathcal{F}_{1} \supseteq \cdots \supseteq \mathcal{F}_{n-1}=\mathcal{F}
$$

where $\mathcal{F}$ and $\mathcal{F}_{i}$ are defined as in (3.3) and (3.4), respectively. Moreover,

$$
\left.P_{\mathcal{F}_{i-1}} \tilde{T}\right|_{\mathcal{F}_{i-1}}=\left(\left.P_{\mathcal{F}_{i-1}} \tilde{T}_{1}\right|_{\mathcal{F}_{i-1}}, \ldots,\left.P_{\mathcal{F}_{i-1}} \tilde{T}_{n}\right|_{\mathcal{F}_{i-1}}\right),
$$

is a commuting tuple and

$$
\left(\left.P_{\mathcal{F}_{i-1}} \tilde{T}_{j}\right|_{\mathcal{F}_{i-1}}\right)\left(\mathcal{F}_{i-1} \ominus \mathcal{F}_{i}\right) \subseteq \mathcal{F}_{i-1} \ominus \mathcal{F}_{i}
$$

for all $i=2, \ldots, n-1$, and $j=1, \ldots, n$.

We now proceed to estimate a lower bound of $\operatorname{mult}_{\tilde{T} \mid \mathcal{S}}(\mathcal{S})$. Note first that $\operatorname{ran}\left(\tilde{P}_{n-1} \tilde{P}_{n}\right)$ is a joint $\tilde{T}$-invariant subspace and

$$
\mathcal{F}_{1}=\mathcal{S} \ominus \operatorname{ran}\left(\tilde{P}_{n-1} \tilde{P}_{n}\right) .
$$

Then $\mathcal{F}_{1}$ is a $\tilde{T}$-semi invariant subspace, which, by Lemma 2.2, implies that

$$
\operatorname{mult}_{\left.\tilde{T}\right|_{\mathcal{S}}}(\mathcal{S}) \geq \operatorname{mult}_{\left.P_{\mathcal{F}_{1}} \tilde{T}\right|_{\mathcal{F}_{1}}}\left(\mathcal{F}_{1}\right)
$$

Now consider the commuting $n$-tuple $\left.P_{\mathcal{F}_{1}} \tilde{T}\right|_{\mathcal{F}_{1}}=\left(\left.P_{\mathcal{F}_{1}} \tilde{T}_{1}\right|_{\mathcal{F}_{1}}, \ldots,\left.P_{\mathcal{F}_{1}} \tilde{T}_{n}\right|_{\mathcal{F}_{1}}\right)$ on $\mathcal{F}_{1}$. Then by Lemma 3.2 we infer that $\mathcal{F}_{1} \ominus \mathcal{F}_{2}$ is a joint $\left.P_{\mathcal{F}_{1}} \tilde{T}\right|_{\mathcal{F}_{1}}$-invariant subspace of $\mathcal{F}_{1}$. But since $\mathcal{F}_{2}=\mathcal{F}_{1} \ominus\left(\mathcal{F}_{1} \ominus \mathcal{F}_{2}\right)$, it follows again by Lemma 2.2 that

$$
\operatorname{mult}_{\left.P_{\mathcal{F}_{1}} \tilde{T}\right|_{\mathcal{F}_{1}}}\left(\mathcal{F}_{1}\right) \geq \operatorname{mult}_{P_{\mathcal{F}_{2}} \tilde{T} \mid \mathcal{F}_{2}}\left(\mathcal{F}_{2}\right) .
$$

In general, by virtue of Lemma 3.2 , we have

$$
\operatorname{mult}_{\left.P_{\mathcal{F}_{i-1}} \tilde{T}\right|_{\mathcal{F}_{i-1}}}\left(\mathcal{F}_{i-1}\right) \geq \operatorname{mult}_{\left.P_{\mathcal{F}_{i}} \tilde{T}\right|_{\mathcal{F}_{i}}}\left(\mathcal{F}_{i}\right)
$$

for all $i=2, \ldots, n-1$, and hence

$$
\operatorname{mult}_{\left.\tilde{T}\right|_{\mathcal{S}}}(\mathcal{S}) \geq \operatorname{mult}_{\left.P_{\mathcal{F}_{1}} \tilde{T}\right|_{\mathcal{F}_{1}}}\left(\mathcal{F}_{1}\right) \geq \cdots \geq \operatorname{mult}_{P_{\mathcal{F}_{n-1}} \tilde{T}_{\mathcal{F}_{n-1}}}\left(\mathcal{F}_{n-1}\right)=\operatorname{mult}_{P_{\mathcal{F}} \tilde{T}||_{\mathcal{F}}}(\mathcal{F}),
$$

where $($ see $(3.3))$

$$
\mathcal{F}=\operatorname{ran} X_{1} \oplus \operatorname{ran}\left(\tilde{Q}_{1} X_{2}\right) \oplus \cdots \oplus \operatorname{ran}\left(\tilde{Q}_{1} \cdots \tilde{Q}_{n-1} X_{n}\right),
$$

and $X_{i}=\tilde{P}_{i} \tilde{Q}_{i+1} \cdots \tilde{Q}_{n}, i=1, \ldots, n$. We summarize the above discussion in the following theorem:

Theorem 3.3. Let $T_{1}, \ldots, T_{n}$ be bounded linear operators on Hilbert spaces $\mathcal{H}_{1}, \ldots, \mathcal{H}_{n}$, respectively. If $\mathcal{Q}_{i}$ is a $T_{i}^{*}$-invariant closed subspace of $\mathcal{H}_{i}, i=1, \ldots, n$, and

$$
\mathcal{S}=\left(\mathcal{Q}_{1} \otimes \cdots \otimes \mathcal{Q}_{n}\right)^{\perp}
$$

then

$$
\operatorname{mult}_{\left.\tilde{T}\right|_{\mathcal{S}}}(\mathcal{S}) \geq \operatorname{mult}_{\left.P_{\mathcal{F}} \tilde{T}\right|_{\mathcal{F}}}(\mathcal{F})
$$




\section{Additivity of multiplicities}

We now proceed to prove the reverse inequality in Theorem 3.3. We start with a simple but useful lemma.

Lemma 4.1. Let $\left(A_{1}, \ldots, A_{n}\right)$ be an $n$-tuple of bounded linear operators on a Hilbert space $\mathcal{H}$. If $G$ is a subset of $\mathcal{H}$ and $\left(\lambda_{1}, \ldots, \lambda_{n}\right) \in \mathbb{C}^{n}$, then

$$
[G]_{\left(A_{1}, \ldots, A_{n}\right)}=[G]_{\left(A_{1}-\lambda_{1} I_{\mathcal{H}}, \ldots, A_{n}-\lambda_{n} I_{\mathcal{H}}\right)} .
$$

Proof. Note that, given $p \in \mathbb{C}\left[z_{1}, \ldots, z_{n}\right]$ there exists $q \in \mathbb{C}\left[z_{1}, \ldots, z_{n}\right]$ such that

$$
\begin{aligned}
p\left(A_{1}, \ldots, A_{n}\right) & =p\left(\left(A_{1}-\lambda_{1} I_{\mathcal{H}}+\lambda_{1} I_{\mathcal{H}}\right), \ldots,\left(A_{n}-\lambda_{n} I_{\mathcal{H}}+\lambda_{n} I_{\mathcal{H}}\right)\right) \\
& =q\left(\left(A_{1}-\lambda_{1} I_{\mathcal{H}}\right), \ldots,\left(A_{n}-\lambda_{n} I_{\mathcal{H}}\right)\right),
\end{aligned}
$$

which implies that

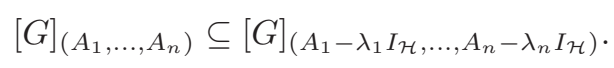

The reverse inclusion follows similarly, and hence the result follows.

Now we return to the problem of rank computation of $\mathcal{S}$ as in Theorem 3.3. From now on, we will use the setting and notation introduced in $\S 3$. Observe that, by (3.3), we have

$$
\mathcal{F}=\mathcal{M}_{1} \oplus \cdots \oplus \mathcal{M}_{n}
$$

where

$$
\mathcal{M}_{i}=\operatorname{ran}\left(\tilde{P}_{i} \prod_{j \neq i} \tilde{Q}_{j}\right) .
$$

By defining $M_{i}=P_{\mathcal{M}_{i}}, i=1, \ldots, n$, one has (see (3.6))

$$
P_{\mathcal{F}}=M_{1} \oplus \cdots \oplus M_{n}
$$

Recall, by virtue of (3.8), that

$$
P_{\mathcal{F}} \tilde{T}_{s} P_{\mathcal{F}}=M_{1} \tilde{T}_{s} M_{1} \oplus \cdots \oplus M_{n} \tilde{T}_{s} M_{n}
$$

for all $s=1, \ldots, n$. And, finally, recall that, by Lemma $3.2, P_{\mathcal{F}} \tilde{T} P_{\mathcal{F}}$ is a commuting tuple on $\mathcal{F}$. The equality in (4.1) implies that

$$
\left(P_{\mathcal{F}} \tilde{T}_{s} P_{\mathcal{F}}\right) \mathcal{M}_{i} \subseteq \mathcal{M}_{i} \quad(s=1, \ldots, n),
$$

that is, $\mathcal{M}_{i}$ is a joint $P_{\mathcal{F}} \tilde{T} P_{\mathcal{F}}$-invariant subspace of $\mathcal{F}$ for all $i=1, \ldots, n$. Then by virtue of (3.9), we have

$$
\left(\left.P_{\mathcal{F}} \tilde{T}\right|_{\mathcal{F}}\right)^{k}=\left.\bigoplus_{i=1}^{n} P_{\mathcal{M}_{i}} \tilde{T}^{k}\right|_{\mathcal{M}_{i}} \quad\left(k \in \mathbb{Z}_{+}^{n}\right)
$$


Now let $G$ be a minimal generating subset of $\mathcal{F}$ with respect to $\left.P_{\mathcal{F}} \tilde{T}\right|_{\mathcal{F}}$. Then

$$
\mathcal{F}=\overline{\operatorname{span}}\left\{\left(\left.P_{\mathcal{F}} \tilde{T}\right|_{\mathcal{F}}\right)^{k}(G): k \in \mathbb{Z}_{+}^{n}\right\} \subseteq \bigoplus_{i=1}^{n}\left(\overline{\operatorname{span}}\left\{\left.P_{\mathcal{M}_{i}} \tilde{T}^{k}\right|_{\mathcal{M}_{i}}(G): k \in \mathbb{Z}_{+}^{n}\right\}\right) \subseteq \mathcal{F}
$$

and so

$$
\mathcal{F}=\bigoplus_{i=1}^{n}\left(\overline{\operatorname{span}}\left\{\left.P_{\mathcal{M}_{i}} \tilde{T}^{k}\right|_{\mathcal{M}_{i}}(G): k \in \mathbb{Z}_{+}^{n}\right\}\right)
$$

Now assume that the point spectrum $\sigma_{p}\left(\left.T_{i}^{*}\right|_{\mathcal{Q}_{i}}\right) \neq \emptyset,\left.T_{i}\right|_{\mathcal{S}_{i}}$ satisfies the generating wandering subspace property, and

$$
\operatorname{dim}\left(\mathcal{S}_{i} \ominus T_{i} \mathcal{S}_{i}\right)<\infty
$$

for all $i=1, \ldots, n$. If we then let $\bar{\alpha}_{i} \in \sigma_{p}\left(\left.T_{i}^{*}\right|_{\mathcal{Q}_{i}}\right)$ and $T_{i}^{*} v_{i}=\bar{\alpha}_{i} v_{i}$ for some non-zero $v_{i} \in \mathcal{Q}_{i}$, then

$$
\mathcal{E}_{i}:=\operatorname{ran}\left(\tilde{P}_{\mathcal{S}_{i} \ominus T_{i} \mathcal{S}_{i}} \prod_{j \neq i} \tilde{P}_{\mathbb{C} v_{j}}\right) \subseteq \mathcal{M}_{i}
$$

and

$$
\operatorname{dim} \mathcal{E}_{i}=\operatorname{dim}\left(\mathcal{S}_{i} \ominus T_{i} \mathcal{S}_{i}\right)=\operatorname{mult}_{T_{i} \mid \mathcal{S}_{i}}\left(\mathcal{S}_{i}\right),
$$

for all $i=1, \ldots, n$. Thus, if we set

$$
\mathcal{E}=\mathcal{E}_{1} \oplus \cdots \oplus \mathcal{E}_{n}
$$

then $\mathcal{E} \subseteq \mathcal{F}$ and

$$
\operatorname{dim} \mathcal{E}=\sum_{i=1}^{n} \operatorname{mult}_{\left.T_{i}\right|_{\mathcal{S}_{i}}}\left(\mathcal{S}_{i}\right)
$$

Fix $i \in\{1, \ldots, n\}$ and define $\left(\lambda_{1}, \ldots, \lambda_{n}\right) \in \mathbb{C}^{n}$ by $\lambda_{j}=0$ if $j=i$ and $\lambda_{j}=\alpha_{j}$ if $j \neq i$. From Lemma 4.1, it follows that

$$
\left[P_{\mathcal{M}_{i}} G\right]_{\left.P_{\mathcal{M}_{i}} \tilde{T}\right|_{\mathcal{M}_{i}}}=\left[P_{\mathcal{M}_{i}} G\right]_{\left(\left.P_{\mathcal{M}_{i}} \tilde{T}_{1}\right|_{\mathcal{M}_{i}}-\lambda_{1} I_{\mathcal{M}_{i}}, \ldots,\left.P_{\mathcal{M}_{i}} \tilde{T}_{n}\right|_{\mathcal{M}_{i}}-\lambda_{n} I_{\mathcal{M}_{i}}\right)}
$$

For simplicity, we denote

$$
\mathcal{G}_{i}=\left[P_{\mathcal{M}_{i}} G\right]_{\left(\left.P_{\mathcal{M}_{i}} \tilde{T}_{1}\right|_{\mathcal{M}_{i}}-\lambda_{1} I_{\mathcal{M}_{i}}, \ldots,\left.P_{\mathcal{M}_{i}} \tilde{T}_{n}\right|_{\mathcal{M}_{i}}-\lambda_{n} I_{\mathcal{M}_{i}}\right)},
$$

in the rest of this section. Also, notice that $\mathbb{C} v_{j} \perp \operatorname{ran}\left(\left.P_{\mathcal{Q}_{j}} T_{j}\right|_{\mathcal{Q}_{j}}-\alpha_{j} I_{\mathcal{Q}_{j}}\right)$ for all $j=1, \ldots, n$, and $\left.\operatorname{ran} T_{i}\right|_{\mathcal{S}_{i}} \perp \mathcal{S}_{i} \ominus T_{i} \mathcal{S}_{i}$, so that

$$
P_{\mathcal{E}_{i}}\left(\left.P_{\mathcal{M}_{i}} \tilde{T}_{j}\right|_{\mathcal{M}_{i}}-\lambda_{j} I_{\mathcal{M}_{i}}\right)=0
$$

for all $j=1, \ldots, n$, and hence

$$
P_{\mathcal{E}_{i}} \mathcal{G}_{i}=P_{\mathcal{E}_{i}}(\overline{\operatorname{span}}\{G\})
$$


On the other hand, since

$$
P_{\mathcal{E}}=\bigoplus_{j=1}^{n} P_{\mathcal{E}_{j}}
$$

and $\mathcal{E}_{j} \subseteq \mathcal{M}_{j}$ for all $j=1, \ldots, n$, it follows that

$$
P_{\mathcal{E}} \mathcal{G}_{i}=P_{\mathcal{E}_{i}} \mathcal{G}_{i}
$$

Hence

$$
\mathcal{E}=P_{\mathcal{E}} \mathcal{F}=P_{\mathcal{E}}\left(\bigoplus_{i=1}^{n}\left[P_{\mathcal{M}_{i}} G\right]_{P_{\mathcal{M}_{i}} \tilde{T} \mid \mathcal{M}_{i}}\right)=\bigoplus_{i=1}^{n} P_{\mathcal{E}_{i}}\left[P_{\mathcal{M}_{i}} G\right]_{\left.P_{\mathcal{M}_{i}} \tilde{T}\right|_{\mathcal{M}_{i}}}
$$

that is

$$
\mathcal{E}=\bigoplus_{i=1}^{n} P_{\mathcal{E}_{i}} \mathcal{G}_{i}=\bigoplus_{i=1}^{n} P_{\mathcal{E}_{i}}(\overline{\operatorname{span}}\{G\})
$$

and so

$$
\mathcal{E}=P_{\mathcal{E}}(\overline{\operatorname{span}}\{G\})
$$

From this it follows easily that

$$
\begin{aligned}
\sum_{i=1}^{n} \operatorname{dim}\left(\mathcal{S}_{i} \ominus T_{i} \mathcal{S}_{i}\right) & =\sum_{i=1}^{n} \operatorname{mult}_{\left.T_{i}\right|_{\mathcal{S}_{i}}}\left(\mathcal{S}_{i}\right) \\
& =\operatorname{dim\mathcal {E}} \\
& \leq \operatorname{dim}(\overline{\operatorname{span}}\{G\}) \\
& =\operatorname{dim}(\operatorname{span}\{G\}) \\
& =\operatorname{mult}_{\left.P_{\mathcal{F}} \tilde{T}\right|_{\mathcal{F}}}(\mathcal{F})
\end{aligned}
$$

where the last equality follows from the minimality assumption on $G$. Therefore, Theorem 3.3 implies the following:

Theorem 4.2. Assume the setting of Theorem 3.3. If $\mathcal{S}_{i}$ satisfies the generating wandering subspace property with respect to $\left.T_{i}\right|_{\mathcal{S}_{i}}$ and $\left.T_{i}^{*}\right|_{\mathcal{Q}_{i}}$ has non-empty point spectrum for all $i=1, \ldots, n$, then

$$
\text { mult }_{\left.\tilde{T}\right|_{\mathcal{S}}}(\mathcal{S}) \geq \sum_{i=1}^{n} \text { mult }_{\left.T_{i}\right|_{\mathcal{S}_{i}}}\left(\mathcal{S}_{i}\right)=\sum_{i=1}^{n} \operatorname{dim}\left(\mathcal{S}_{i} \ominus T_{i} \mathcal{S}_{i}\right)
$$

To proceed further, we note, by Lemma 3.1 (or, more specifically (3.2)), that

$$
\mathcal{S}=\sum_{i=1}^{n} \operatorname{ran} \tilde{P}_{i}
$$

In addition, let us assume that $\operatorname{mult}_{T_{i}}\left(\mathcal{H}_{i}\right)=1, i=1, \ldots, n$. Then

$$
\operatorname{mult}_{\left.\tilde{T}\right|_{\mathcal{S}}}(\mathcal{S}) \leq \sum_{i=1}^{n} \operatorname{mult}_{\left.T_{i}\right|_{\mathcal{S}_{i}}}\left(\mathcal{S}_{i}\right)
$$

Therefore, by Theorem 4.2, we have the main theorem of this paper as: 
Theorem 4.3. Let $\mathcal{H}_{1}, \ldots, \mathcal{H}_{n}$ be Hilbert spaces, let $T_{i} \in \mathcal{B}\left(\mathcal{H}_{i}\right)$, and let $\mathcal{Q}_{i}$ be a $T_{i}^{*}$ invariant closed subspace of $\mathcal{H}_{i}, i=1, \ldots, n$. Assume that $\left.T_{i}\right|_{\mathcal{Q}_{i}^{\perp}} \in \mathcal{B}\left(\mathcal{Q}_{i}^{\perp}\right)$ satisfies the generating wandering subspace property, $\left.T_{i}^{*}\right|_{\mathcal{Q}_{i}}$ has non-empty point spectrum and that mult $_{T_{i}}\left(\mathcal{H}_{i}\right)=1$ for all $i=1, \ldots, n$. Then

$$
\operatorname{mult}_{\left.\tilde{T}\right|_{\left(\mathcal{Q}_{1} \otimes \cdots \otimes \mathcal{Q}_{n}\right)^{\perp}}}\left(\mathcal{Q}_{1} \otimes \cdots \otimes \mathcal{Q}_{n}\right)^{\perp}=\sum_{i=1}^{n} \operatorname{mult}_{\left.T_{i}\right|_{\mathcal{Q}_{i}^{\perp}}}\left(\mathcal{Q}_{i}^{\perp}\right) .
$$

\section{Applications and concluding remarks}

In this section, we complement the main theorem, Theorem 4.3, by some concrete examples and final remarks.

We first explain the notion of zero-based invariant subspaces of reproducing kernel Hilbert spaces. Let $k: \mathbb{D} \times \mathbb{D} \rightarrow \mathbb{C}$ be a positive definite kernel. For each fixed $w \in \mathbb{D}$, let $z \mapsto k(z, w)$ is analytic on $\mathbb{D}$. Suppose $\mathcal{H}_{k} \subseteq \mathcal{O}(\mathbb{D})$ is the reproducing kernel Hilbert space corresponding to the kernel $k$ and $M_{z}$, the multiplication operator by the coordinate function $z$, on $\mathcal{H}_{k}$ is bounded. Let us further assume that

$$
\operatorname{ker}\left(M_{z}^{*}-\lambda I_{\mathcal{H}_{k}}\right)=\mathbb{C} k(\cdot, \lambda) \quad(\lambda \in \mathbb{D}) .
$$

Here $k(\cdot, \lambda)$, for $\lambda \in \mathbb{D}$, denotes the kernel function $z \mapsto k(z, \lambda)$ on $\mathbb{D}$.

A reproducing kernel Hilbert space that satisfies all the properties listed above is called a regular reproducing kernel Hilbert space.

It is easy to see that the Dirichlet space, the Hardy, the unweighted Bergman space and the weighted Bergman spaces over $\mathbb{D}$ are regular reproducing kernel Hilbert spaces.

Suppose $\mathcal{H}_{k}$ is a regular reproducing kernel Hilbert space. A closed subspace $\mathcal{S} \subseteq \mathcal{H}_{k}$ is called zero-based invariant subspace if there exists $\lambda \in \mathbb{D}$ such that $f(\lambda)=0$ for all $f \in \mathcal{S}$ and $z \mathcal{S} \subseteq \mathcal{S}$.

Now let $\mathcal{H}_{k}$ be a regular reproducing kernel Hilbert space, and let $\mathcal{Q}$ be an $M_{z}^{*}$-invariant closed subspace of $\mathcal{H}_{k}$. Suppose $\lambda \in \mathbb{D}$. Then $M_{z}^{*} f=\bar{\lambda} f$ for some non-zero $f \in \mathcal{Q}$ if and only if $f=c k(\cdot, \lambda)$ for some non-zero scalar $c \in \mathbb{C}$. On the other hand, since

$$
\langle g, k(\cdot, \lambda)\rangle=g(\lambda) \quad\left(g \in \mathcal{H}_{k}\right)
$$

it follows that $k(\cdot, \lambda) \in \mathcal{Q}$ if and only if $g(\lambda)=0$ for all $g \in \mathcal{Q}^{\perp}$. We have therefore proved the following:

Proposition 5.1. Let $\mathcal{H}_{k}$ be a regular reproducing kernel Hilbert space, and let $\mathcal{Q}$ be a closed $M_{z}^{*}$-invariant subspace of $\mathcal{H}_{k}$. Then $\left.M_{z}^{*}\right|_{\mathcal{Q}}$ has non-empty point spectrum if and only if $\mathcal{Q}^{\perp}$ is a zero-based invariant subspace of $\mathcal{H}_{k}$.

As an immediate corollary of Theorem 4.3, we have now:

Corollary 5.2. Let $\mathcal{H}_{k_{i}}$ be a regular reproducing kernel Hilbert space, mult $_{M_{z}}\left(\mathcal{H}_{k_{i}}\right)=$ 1 , and let $\mathcal{Q}_{i}$ be a proper closed $M_{z}^{*}$-invariant subspace of $\mathcal{H}_{k_{i}}, i=1, \ldots, n$. If $\mathcal{Q}_{i}^{\perp}$ is a 
zero-based invariant subspace of $\mathcal{H}_{k_{i}}$ such that

$$
\operatorname{dim}\left(\mathcal{Q}_{i}^{\perp} \ominus z \mathcal{Q}_{i}^{\perp}\right)<\infty,
$$

for all $i=1, \ldots, n$, then

$$
\operatorname{mult}_{\left.M_{z}\right|_{\left(\mathcal{Q}_{1} \otimes \cdots \otimes \mathcal{Q}_{n}\right)^{\perp}}}\left(\mathcal{Q}_{1} \otimes \cdots \otimes \mathcal{Q}_{n}\right)^{\perp}=\sum_{i=1}^{n}\left(\operatorname{mult}_{\left.M_{z}\right|_{\mathcal{Q}_{i}^{\perp}}}\left(\mathcal{Q}_{i}^{\perp}\right)\right)=\sum_{i=1}^{n} \operatorname{dim}\left(\mathcal{Q}_{i}^{\perp} \ominus z \mathcal{Q}_{i}^{\perp}\right) .
$$

Now let $\mathcal{H}_{k_{i}}$ be the Hardy space or the Dirichlet space over $\mathbb{D}$, and let $\mathcal{Q}_{i}$ be a non-zero shift co-invariant (that is, $M_{z}^{*}$-invariant) subspace of $\mathcal{H}_{k_{i}}$. By $[3,16],\left.M_{z}\right|_{\mathcal{Q}_{i}^{\perp}}$ satisfies the generating wandering subspace property and the dimension of the generating wandering subspace is one, that is

$$
\operatorname{dim}\left(\mathcal{Q}_{i}^{\perp} \ominus z \mathcal{Q}_{i}^{\perp}\right)=1,
$$

for all $i=1, \ldots, n$. Then, in view of Theorem 4.3 (and [19]) we have the following:

Corollary 5.3. Let $\mathcal{H}_{k_{i}}, i=1, \ldots, n$, denote either the Hardy space or the Dirichlet space over $\mathbb{D}$. Suppose $\mathcal{Q}_{i}$ is a proper closed $M_{z}^{*}$-invariant subspaces of $\mathcal{H}_{k_{i}}, i=1, \ldots, n$. If $\mathcal{Q}_{i}^{\perp}$ is a zero-based $M_{z}$-invariant subspace of $\mathcal{H}_{k_{i}}, i=1, \ldots, n$, then,

$$
\text { mult }_{\left.M_{z}\right|_{\left(\mathcal{Q}_{1} \otimes \cdots \otimes \mathcal{Q}_{n}\right)^{\perp}}}\left(\mathcal{Q}_{1} \otimes \cdots \otimes \mathcal{Q}_{n}\right)^{\perp}=n .
$$

A similar argument and the generating wandering subspace property of shift invariant subspaces of the Bergman space [1] yields the following:

Corollary 5.4. Let $\mathcal{H}_{k_{i}}, i=1, \ldots, n$, be the Dirichlet space, the Bergman space or the Hardy space over $\mathbb{D}$. Let $\mathcal{Q}_{i}, i=1, \ldots, n$, be proper closed shift co-invariant subspaces of $\mathcal{H}_{k_{i}}$. If $\mathcal{Q}_{i}^{\perp}$ is a zero based $M_{z}$-invariant subspace of $\mathcal{H}_{k_{i}}$ and

$$
\operatorname{dim}\left(\mathcal{Q}_{i}^{\perp} \ominus z \mathcal{Q}_{i}^{\perp}\right)<\infty,
$$

for all $i=1, \ldots, n$, then

$$
\operatorname{mult}_{\left.M_{z}\right|_{\left(\mathcal{Q}_{1} \otimes \cdots \otimes \mathcal{Q}_{n}\right)^{\perp}}}\left(\mathcal{Q}_{1} \otimes \cdots \otimes \mathcal{Q}_{n}\right)^{\perp}=\sum_{i=1}^{n}\left(\operatorname{mult}_{\left.M_{z}\right|_{\mathcal{Q}_{i}^{\perp}}}\left(\mathcal{Q}_{i}^{\perp}\right)\right)=\sum_{i=1}^{n} \operatorname{dim}\left(\mathcal{Q}_{i}^{\perp} \ominus z \mathcal{Q}_{i}^{\perp}\right) .
$$

Note that the generating wandering subspace assumption in Corollary 5.4 ensures that (see Proposition 2.1)

$$
\operatorname{mult}_{\left.M_{z}\right|_{\mathcal{Q}_{i}^{\perp}}}\left(\mathcal{Q}_{i}^{\perp}\right)<\infty,
$$

for all $i=1, \ldots, n$. At present, it is not very clear whether the generating wandering subspace assumption can be replaced by finite multiplicity property. Our methods rely heavily on the assumption that the invariant subspaces are zero-based and satisfies the generating wandering subspace property.

Acknowledgments. The research of the first-named author is supported by the Mathematical Research Impact Centric Support, MATRICS (MTR/2019/000640), by 
SERB, Government of India. The second-named author is supported in part by the Mathematical Research Impact Centric Support, MATRICS (MTR/2017/000522), and Core Research Grant (CRG/2019/000908), by SERB (DST), and NBHM (NBHM/R.P.64/2014), Government of India. The research of the third-named author is supported by DST-INSPIRE Faculty Fellowship No. - DST/INSPIRE/04/2019/000769. He would also like to acknowledge in part the NBHM postdoctoral fellowship NBHM PDF No. 0204/52/2019/R/D-II/342 and Indian Statistical Institute Bangalore, for a visiting scientist position under which part of the work was done.

\section{References}

1. A. Aleman, S. Richter and C. Sundberg, Beurling's theorem for the Bergman space, Acta Math. 177 (1996), 275-310.

2. C. Apostol, H. Bercovici, C. Foias and C. Pearcy, Invariant subspaces, dilation theory, and the structure of the predual of a dual algebra I, J. Funct. Anal. 63 (1985), 369-404.

3. A. Beurling, On two problems concerning linear transformations in Hilbert space, Acta Math. 81 (1949), 239-255.

4. A. Chattopadhyay, B. K. Das and J. Sarkar, Star-generating vectors of Rudin's quotient modules, J. Funct. Anal. 267 (2014), 4341-4360.

5. A. Chattopadhyay, B. K. Das and J. Sarkar, Rank of a co-doubly commuting submodule is 2, Proc. Amer. Math. Soc. 146 (2018), 1181-1187.

6. R. Douglas And R. YAng, Operator theory in the Hardy space over the bidisk (I), Integral Equations Operator Theory 38(2) (2000), 207-221.

7. X. FAng, Additive invariants on the Hardy space over the polydisc, J. Funct. Anal. $\mathbf{2 5 3}$ (2007), 359-372.

8. H. Hedenmalm, An invariant subspace of the Bergman space having the codimension two property, J. Reine Angew. Math. 443 (1993), 1-9.

9. H. Hedenmalm, B. Korenblum and K. Zhu, Theory of Bergman spaces, Graduate Texts in Mathematics, Volume 199 (Springer-Verlag, New York, 2000).

10. H. Hedenmalm, S. Richter And K. Seip, Interpolating sequences and invariant subspaces of given index in the Bergman spaces, J. Reine Angew. Math. 477 (1996), $13-30$.

11. K. J. IzUChi, K. H. IzUChi AND Y. IzUChI, Blaschke products and the rank of backward shift invariant subspaces over the bidisk, J. Funct. Anal. 261(6) (2011), 1457-1468.

12. K. J. IzUChi, K. H. IzUCHI AND Y. IzUChI, Ranks of invariant subspaces of the Hardy space over the bidisk, J. Reine Angew. Math. 659 (2011), 101-139.

13. K. J. IzUChI, K. H. IzUChI AND Y. IzUChI, Ranks of backward shift invariant subspaces of the Hardy space over the bidisk, Math. Z. 274 (2013), 885-903.

14. G. POPESCU, Euler characteristic on noncommutative polyballs, J. Reine Angew. Math. $\mathbf{7 2 8}$ (2017), 195-236.

15. G. POPESCU, Invariant subspaces and operator model theory on noncommutative varieties, Math. Ann. 372 (2018), 611-650.

16. S. Richter, Invariant subspaces of the Dirichlet shift, J. Reine Angew. Math. 386 (1988), 205-220.

17. W. Rudin, Function theory in polydiscs (New York, Benjamin, 1969).

18. J. SARKAR, Jordan blocks of $H^{2}\left(\mathbb{D}^{n}\right)$, J. Operator Theory 72 (2014), 371-385.

19. A. Tomerlin, Products of Nevanlinna-Pick kernels and operator colligations, Integr. Equ. Oper. Theory 38(3) (2000), 350-356. 